\title{
Zur Kenntnis der menschlichen Gallensteine.
}

\section{Von}

E. Salkowski.

(Ans der chemischen Abteilung des Pathologischen Instituts der Universität Berlin.) (Der Redaktion zugegangen am 6. August 1916.)

Vor kurzem ${ }^{1}$ ) habe ich mitgeteilt, daß ich an autolysierter Rindergalle eine Ausscheidung von palmitinsaurem Kalk in nicht unbeträchtlicher Quantität beobachtet habe, eine Erscheinung, die auf die Spaltung von Choleinsäure bezw. Glykocholeinsäure und Taurocholeinsäure im Sinne von Wieland und Sorge $e^{2}$ ) zurückzuführen sein möchte, und habe der Möglichkeit gedacht, daß diese Spaltung auch durch Bacterium coli oder andere Darmbakterien stattfinden und der Ausgangspunkt bei der Bildung von Gallensteinen beim Menschen sein könnte. Es waren also Gallensteine auf etwaigen Gehalt an Calciumpalmitat zu untersuchen. Hiezu dienten in drei Versuchen je $50 \mathrm{~g}$ menschliche Gallensteine, durch ein feines Drahtnetz gesiebt, und zwar ein Gemisch von Cholesterinsteinen und Farbstoffsteinen. Da der Gang der Untersuchung in den drei Versuchen nicht ganz derselbe war, sich außerdem im Laufe der Arbeit noch ein anderer Gesichtspunkt ergab, beschreibe ich sie einzeln.

I.

Das Gallensteinpulver wurde zuerst mit etwa der zehnfachen Quantität Äther 2 Stunden auf der Schüttelmaschine geschüttelt, abfiltriert, das rückständige Pulver zur Entfernung von Resten von Cholesterin 10 Stunden lang im Soxhlet mit

1) Diese Zeitschr., Bd. 97, S. 210 (1916).

2) Diese Zeitschr., Bd.97, S.3. 
etwa $200 \mathrm{ccm}$ Äther extrahiert, die beiden Auszüge vereinigt. Das Volumen betrug nach Zusatz von etwas Äther $650 \mathrm{ccm} .{ }^{1}$ ) $100 \mathrm{ccm}$ lieferten beim Abdestillieren, Verdampfen und Veraschen nur $0,0016 \mathrm{~g}$ in Salzsäure lösliche Asche, die sich schwach kalkhaltig erwies, aufs Ganze berechnet also 0,010 g. In den Ätherauszug war also, wie zu erwarten, nur eine minimale Quantität eines Calciumsalzes übergegangen.

Das rückständige Gallensteinpulver wurde 10 Stunden lang im Soxhlet mit Alkohol absol. ausgezogen. Der beim Verdunsten des Alkoholauszuges erhaltene Rückstand wog $0,4614 \mathrm{~g}$. Er konnte möglicherweise aus Calciumpalmitat bestehen oder solches enthalten. Zur Untersuchung darauf wurde er in der Reibschale mit Salzsäure und Äther gut durchgearbeitet, das Ganze in einen Scheidetrichter übergeführt und mit Äther gut durchgeschüttelt. Die abgetrennte salzsaure Flüssigkeit erwies sich kalkhaltig.

Der Ätherauszug wurde zur Entfernung der anhängenden Salzsäure nochmals mit Wasser durchgeschüttelt; beim Verdunsten des durch ein trockenes Filter filtrierten Ätherauszuges wurde ein krystallinisch erstarrender, jedoch weicher Rückstand im Gewicht von $0,167 \mathrm{~g}$ erhalten, der sich in $\mathrm{Na}_{2} \mathrm{CO}_{3}$ Lösung unter Entwicklung von $\mathrm{CO}_{2}$ klar löste. Die Lösung wurde mit Salzsäure gefällt, die Fällung abfiltriert, mit Wasser gut gewaschen, an der Luft getrocknet, das Filter mit der anhaftenden Substanz mit Petroleumäther behandelt. Dabei ging nur wenig in Lösung, es konnte also nur wenig Palmitinsäure vorhanden sein. Die Petrolätherlösung hinterließ beim Verdunsten nur eine sehr kleine Quantität eines farblosen krystallinischen Rückstandes vom Schmelzpunkt 57-58 ${ }^{\circ}$, der sich in $\mathrm{Na}_{2} \mathrm{CO}_{3}$ beim Erwärmen löste; die Lösung erstarrte beim Erkalten zu Seifenleim von charakteristischer Beschaffenheit. Damit kann man wohl Palmitinsäure im Gemisch mit etwas Stearinsäure als nachgewiesen ansehen, indessen ist die Quantität für $50 \mathrm{~g}$ Gallensteine so gering, daß sie für die Gallensteinbildung schwerlich in Betracht kommt; außerdem

1) Der Ätherauszug war schwach gelb gefärbt, wurde beim Stehenlassen in einigen Tagen farblos. 
ist nicht bestimmt nachgewiesen, daß sie als Calciumsalz vorhanden war.

Es war nun denkbar, daß etwa in dem Gallensteinpulver vorhandenes Calciumpalmitat von dem Alkohol nur unvollständig aufgenommen war; ich mußte also nach einem besseren Lösungsmittel für dieses suchen. Ein solches fand ich in der Buttersäure (normale). Buttersäure löst palmitinsauren Kalk beim Erhitzen spielend leicht auf, so reichlich, daß die Lösung beim Erkalten einen Krystallbrei bildet, der sich leicht in Alkohol löst. Um aus dieser Lösung palmitinsauren Kalk zu erhalten, braucht man die Lösung nur in Wasser zu gießen: es scheidet sich sofort oder nach einigem Stehen palmitinsaurer Kalk ab. Der abfiltrierte und gut ausgewaschene Niederschlag liefert beim Behandeln mit Salzsäure und Äther Palmitinsäure vom richtigen Schmelzpunkt. ${ }^{1}$ )

Die Untersuchung des nach dem Ausziehen mit Alkohol gebliebenen Gallensteinpulvers - mit Hilfe des Buttersäureverfahrens - hatte ein fast negatives Resultat und es wurden nur $0,0150 \mathrm{~g}$ Substanz erhalten, die möglicherweise Palmitinsäure hätte sein können, die Einzelheiten haben daher kein Interesse.

Ich kehre zu dem Anteil der Säuren zurück, der sich in Petroläther nicht gelöst hatte. Derselbe stellte nach dem Abdunsten des anhängenden Petroläthers eine harzartige spröde Masse dar. Es war beinahe selbstverständlich darin eine Gallensäure zu sehen, umsomehr als die alkalische Lösung desselben - in einer Probe untersucht - schäumte und bitter schmeckte. Auffallenderweise aber gab dieselbe die Petten-

1) Es sei noch bemerkt, daß dieses Verhalten nicht zur Trennung von Cholesterin benutzt werden kann, denn auch dieses löst sich, wie nach dem Verhalten zu Eisessig zu erwarten war - buttersaures Cholesterin ist noch nicht beschrieben - reichlich in Buttersäure. - Leider zu spät fand ich, daß man zum Lösen von Calciumpalmitat statt Buttersäure ebensogut Eisessig nehmen kann, was natürlich vorzuziehen ist. $\mathrm{Da} B$ ich auf Buttersäure verfiel, hängt damit zusammen, daß ich früher bei Untersuchung von Leichenwachs beobachtet hatte, daB die Fettsäuren desselben in klar geschmolzenem Zustand erheblich kalkhaltig sind. Auf den \&Fettcharakter> der Buttersäure kommt es also nicht an. 
kofersche Reaktion nur schwierig und etwas abweichend. Dies erinnerte mich an eine Gallensäure, die ich im Jahr 18921) gelegentlich aus den Fäces eines Hundes erhalten hatte. Der betreffende Hund von $25 \mathrm{~kg}$ Körpergewicht war, mit $450 \mathrm{~g}$ Pferdefleisch und $100 \mathrm{~g}$ Fett pro Tag gefüttert, im Stickstoffgleichgewicht, und hatte zu einem Versuche über den EinfluB der Benzoesäure auf den Eiweißzerfall gedient. Da bei dem Ort der Publikation nicht anzunehmen ist, daB diese Beobachtung zur Kenntnis der speziellen Fachgenossen gekommen ist, soviel ich sehen kann, ist sie auch nicht in den Malyschen Jahresbericht übergegangen -, sei es mir gestattet, die damaligen Beobachtungen hier wörtlich anzuführen. Es heißt 1. c. S. 34: -Nach dem Trocknen über Schwefelsäure zeigte die auf dem angegebenen Wege ${ }^{2}$ ) dargestellte neue Substanz folgende Eigenschaften.

1. Sie stellt ein kreidig weißes Pulver resp. Bröckel dar, ganz unlöslich in Wasser, anscheinend auch in heißem, leicht löslich in Alkohol. Die alkoholische Lösung gibt, eingetrocknet, einen amorphen Rückstand; versetzt man jedoch die in einer Glasschale befindliche Lösung mit etwas Wasser, so scheidet sich beim Stehen ein seidenglänzender Niederschlag aus, welcher, mikroskopisch untersucht, aus Büscheln radiär gruppierter lanzettförmiger Blättchen besteht, oder - bei sehr schneller Ausscheidung durch Zusatz von viel Wasser - aus Nadeln. Die Substanz ist unlöslich oder jedenfalls sehr schwer löslich in Äther, leicht bei nachträglichem Zusatz von ein wenig Alkohol.

2. Der Schmelzpunkt liegt bei $158^{\circ}$.

3. Beim Erhitzen auf dem Platinblech schmilzt die Säure unter Verbreitung aromatischer, terpentinartig riechender Dämpfe, verbrennt dann mit leuchtender Flamme, ohne Asche zu hinterlassen.

4. In konzentrierter Schwefelsäure löst sich die Substanz leicht; die Lösung ist gelb, im auffallenden Licht grün.

i) Festschrift für L e yden: Internationale Beiträge zur inneren Medizin. Bd. II, Verlag von A. Hirschwald, 1902. - Meine Beobachtung stammt aus 1892 .

2) Der hier übergangen werden kann. 
5. Sie löst sich leicht unter Entwicklung von Kohlensäure in Natriumcarbonatlösung. Die Lösung schmeckt bitter, gibt die Pettenk of ersche Reaktion nur schwierig. Am besten erhielt man diese Reaktion, wenn man $\mathrm{zu}$ der Lösung in konzentrierter Schwefelsäure vorsichtig etwas sehr verdünnte Rohrzuckerlösung hinzutropft: die Färbung ist nicht kirschrot, sondern mehr bordeauxrot; gießt man jedoch ein wenig der Reaktionsflüssigkeit in Eisessig ein, so erhält man eine intensiv und rein violett gefärbte Lösung. Die Lösung zeigt keinen Absorptionsstreifen im Spektrum.

6. Die Substanz enthält Spuren von Stickstoff, vermutlich als Verunreinigung.

Nach alledem ist die erhaltene Säure, die bei Stoffwechseluntersuchungen sicher zum Teil als «Rohfett * erscheint, wohl ohne Zweifel den Gallensäuren hinzuzurechnen. Von der Cholsäure (Cholalsäure) unterseheidet sie sich durch ihr Verhalten zu Jodjodkaliumlösung.

Versetzt man eine alkoholische Lösung von Cholsäure mit Wasser, dann mit Jodjodkaliumlösung, so entsteht, entsprechend den Angaben von Mylius, ein bräunlich gefärbter Brei, welcher, abfiltriert oder in dünner Schicht auf einem Uhrglas eingetrocknet, in auffallendem Licht braungelb, metallisch glänzend (bronzefarben) erscheint, in durchfallendem Licht indigoblau. Die Lösung der in Rede stehenden Säure zeigte diese Reaktion nicht; es scheidet sich vielmehr bei Wasser- und Jodzusatz ein weißer Niederschlag aus, welcher aus mikroskopischen Nadeln besteht.»

Eine weitere Untersuchung hat damals nicht stattgefunden; sie hätte bei der noch übrig gebliebenen Quantität $=0,430 \mathrm{~g}$ bei den damaligen Hilfsmitteln auch schwerlich zu einem entscheidenden Resultat geführt. Leider ist das Präparat im Laufe der dazwischen liegenden 24 Jahre abhanden gekommen.

Die vorliegende Gallensäure zeigte nun mit der damals beobachteten eine sehr weitgehende Übereinstimmung. Unter denselben Bedingungen wie jene war sie an den Rändern der Lösung zur Krystallisation zu bringen, während ein Teil der Lösung amorph eintrocknete. Die Krystalle (mikroskopisch) 
waren gleichfalls lanzettförmig bezw. bildeten abgerundete rhombische Tafeln, d. h. abgerundet in bezug auf die Winkel der Längsseiten, während dieSpitzenwinkel gut ausgebildet waren.

Der Schmelzpunkt lag bei $158^{\circ}$ nach vorhergehender Erweichung, also unscharf.

Bezüglich der Punkte 4 und 5, sowie bezüglich des Verhaltens zu Jodjodkaliumlösung stimmt die vorliegende Säure mit der damals heobachteten völlig überein.

Um zu sehen, ob das mit Äther und Alkohol erschöpfte Gallensteinpulver vielleicht noch in diesen Lösungsmitteln unlösliche Palmitinsäureverbindungen enthielt, wurde es mit Salzsäure und reichlichen NIengen Äther behandelt. Die gesamten Ätherauszüge hinterließen nur 0,01 orangegefärbten Rückstand, der sich etwas trüb in Natriumcarbonatlösung löste. Die Lösung gab Gallenfarbstoffreaktion.

II.

Das Gallensteinpulver wurde ebenso wie bei der ersten Untersuchung durch Schütteln mit Äther von dem größten Teil des Cholesterins befreit, dann aber, um etwa vorhandene wasserlösliche Gallenbestandteile, die mechanisch anhaften mochten, zu entfernen, mit Wasser ausgekocht, filtriert. Die orangegefärbte Lösung lieferte beim Eindampfen zur Trockne einen fast schwarzen, glänzenden, harten Überzug in der Schale, der abgekratzt, gepulvert und im Soxhlet mit Alkohol extrahiert wurde. Der Alkoholauszug lieferte beim Verdampfen $0,346 \mathrm{~g}$ in Wasser trüb löslichen Rückstand, der nicht weiter untersucht ist.

Das rückständige Gallensteinpulver wurde nach gutem Trocknen 10 Stunden im Soxhlet mit Äther ausgezogen. Der Äther hinterließ $0,245 \mathrm{~g}$ eines nach Cholesterin aussehenden Rückstandes, der auch Cholesterinreaktion gibt. Er wird mit $\mathrm{Na}_{2} \mathrm{CO}_{3}$-Lösung behandelt, das Filtrat - die Filtration erfolgte sehr zögernd - gibt mit Salzsäure angesäuert weiße Trübung, allmählich weiße, mikroskopisch amorph erscheinende Ausscheidung. Die Quantität derselben nach dem Ausaschen und Trocknenlassen des Filters war so gering, daß nur leichte 
Schmelzbarkeit und Bildung eines Fettflecks auf Papier nachgewiesen werden konnte.

Das mit Äther erschöpfte Gallensteinpulver wurde mit Alkohol ausgezogen. Der Alkoholauszug hinterließ beim Verdunsten nur 0,1310 Rückstand. Dieser wurde in der Wärme mit Buttersäure behandelt, Alkohol hinzugegeben, filtriert, das Filtrat in Wasser gegossen, die sich langsam absetzende Fällung abfiltriert, gut ausgewaschen, getrocknet, dann auf dem Filter mit heißem Alkohol behandelt. Die alkoholische Lösung hinterließ beim Verdunsten 0,0464 Rückstand, der wohl nur aus den Calciumsalzen von Palmitinsäure und Gallensäure bestehen konnte. Er wurde mit Salzsäure und Äther behandelt, der Äther verdunstet, der Rückstand mit Petroläther behandelt. Beim Verdunsten desselben blieben 0,0181 eines sofort krystallinisch erstarrenden Rückstandes vom Schmelzpunkt 57 bis $58^{\circ}$, der sich in $\mathrm{Na}_{2} \mathrm{CO}_{3}$-Lösung löste, mit demselben Seifenleim bildend. Das vom Petroläther nicht Gelöste hatte denselben Charakter, wie in Versuch I und gab wie dieses Gallensäurereaktion.

\section{III.}

$50 \mathrm{~g}$ Gallensteinpulver wurden, wie bisher, erschöpfend mit Äther behandelt, die vereinigten Ätherauszüge zur Untersuchung auf etwa vorhandene freie Säuren mit $100 \mathrm{~cm} \mathrm{n/10-}$ Natronlauge geschüttelt. Dies geschah im Hinblick auf die Auffindung freier Desoxycholsäure von W. Küster, ${ }^{1}$ ) einer neuen «Lithocholsäure genannten Gallensäure von Hans Fischer ${ }^{2}$ ) und die Auffindung von Stearinsäure und Choleinsäure von Hans Fischer und P. Meyer. ${ }^{3}$ ) Alle diese Befunde beziehen sich auf Rindergallensteine. Erst nach tagelangem Stehen trat eine Scheidung der Schichten ein bis auf eine etwa $1 / 2 \mathrm{~cm}$ hohe Zwischenschicht, die nicht berücksichtigt wurde.

Die abgetrennte wässerige Lösung reagierte noch stark alkalisch, es war àiso nur sehr wenig Säure aufgenommen resp.

1) Diese Zeitschr., Bd. 69, S. 463.

2) Diese Zeitschrift, Bd. 73, S. 277.

3) Diese Zeitschr., Bd. 76, S. 96. 
vorhanden gewesen. Die Lösung wurde, nachdem seine trübe Beschaffenheit durch Filtrieren beseitigt war, bis auf wenige Kubikzentimeter eingedampft, dabei schied sich ein gelblich gefärbter Kuchen aus, ein Verhalten, das lebhaft an das Verlalten alkalischer Gallensüurelösungen erinnerte. Beim Zusatz von Salzsïureunter leichtem Erwärmen bildet sich hieraus eine krümlige, körnige Masse. Sie wurde abfiltriert, gewaschen, an der Luft getrocknet und ohne Erwärmen mit Petroleumäther behandelt. Der Petroleumätherauszug hinterlieb $0,0539 \mathrm{~g}$ sofort krystallinisch erstarrenden Rückstand vom Schmelzpunkt 58-590, der sich in $\mathrm{Na}_{2} \mathrm{CO}_{3}$-Lösung unter Bildung von Seifenleim löste.

Das von Petroleumäther nicht Gelöste wog $0,3 \mathrm{C} 7 \mathrm{~g}$. Es wurde nochmals mit Petroleumäther behandelt. Der Verdampfungsrückstand des Auszuges erwies sich wiederum als: Palmitinsäure mit einem Gehalt von Stearinsäure.

Das von Petroläther nicht Gelöste wurde in Eisessig gelöst, die filtrierte essigsaure Lösung mit etwas Wasser versetzt, dann sich selbst überlassen. Die in mikroskopischen Nadeln auskrystallisierte Substanz, abfiltriert, gewaschen und an der Luft getrocknet, zeigte folgende Eigenschaften:

1. Die Lösung in Schwefelsäure war gelb mit grüner Fluorescenz.

2. Die Pettenk ofersche Probe war positiv, die Nüance etwas abweichend, mehr bordeauxrot als purpurrot. Hierzu möchte ich indessen bemerken, daß dies auch bei der Cholsäure, wenn man sehr geringe Mengen verwendet, mitunter der Fall ist. Wiederholt habe ich beobachtet, daß die Lösung bei Zusatz von ein wenig Alkohol absol. prächtig purpurfarben wird. Das gilt ebenso für die Cholsäure. Herr Prof. Hans Fischer hat die Freundlichkeit gehabt, mir ein Pröbchen seiner Lithocholsäure zur Verfügung zu stellen, wofür ich demselben auch an dieser Stelle verbindlichst danke; an dieser beobachtete ich genau das gleiche Verhalten. Bei weiterem Verdünnen mit Alkohol absol. ${ }^{1}$ ) erhielt man bei allen 3 Säuren eine zunächst

1) Man darf zum Verdünnen nicht gewöhnlichen ca. $90 \%$ igen Alkohol nehmen, dann kann es vorkommen, daß man statt der purpurfarbenen Lösung eine mißfarbene erhält. Setzt man zu dieser konzentrierte 
purpurfarbene Lösung. Auch bei meiner Säure war bei der spektroskopischen Untersuchung ein starker Absorptionsstreifen bei $\mathrm{F}$ zu konstatieren, wie es $\mathrm{H}$. Fischer für seine Lithocholsäure angibt. Allmählich verblaßt die Lösung, Zusatz konzentrierter Schwefelsäure stellt öfters die Farbe wieder her, ganz konstant sind die Erscheinungen indessen nicht. Besonders haltbare, jedoch mehr rosa als purpurrote Lösung erhielt ich, wenn ich eine alkoholische Lösung meiner Säure mit etwas Rohrzucker und Schwefelsäure versetzte. Sie hielt sich in einem Falle 2 Tage lang unverändert.

3. Die Säure löst sich in Alkohol ziemlich leicht; bei Zusatz von Wasser scheiden sich amorphe Flocken aus, die sich nach einigem Stehen in glänzende Krystallflitter umwandeln. Unter dem Mikroskop erscheinen diese als lange, schmale Prismen mit zerfaserten Endkanten, hie und da zu Rosetten gruppiert.

4. Der Schmelzpunkt der aus Essigsäure krystallisierten Säure wurde bei $179^{\circ}$ gefunden, die aus Alkohol krystallisierte schmolz bei $182^{\circ}$ (völlige Schmelzung).

5. Die aus Essigsäure krỵstallisierte Säure ist gänzlich geschmacklos, die alkoholische mit Wasser verdünnte Lösung zeigt indessen, so lange sie noch milchig getrübt erscheint (vor der Krystallisation), deutlich schwach bittern Geschmack, ebenso die alkalische Lösung.

6. Die Myliussche Jodreaktion verlief bei mehrfachen Proben durchaus negativ, während sie in Kontrollproben mit Cholsäure sofort gelang.

Schwefelsäure, so stellt sich die rote Farbe wieder her, wenigstens teilweise. Das Verhältnis ist dasselbe, wie ich es für die ChloroformSchwefelsäurereaktion auf Cholesterin angegeben habe. Giebt man bei dieser die Chloroformlösung von der Schwefelsäure ab und verdünnt sie mit weiterem Chloroform, so verschwindet die rote Farbe, augenscheinlich infolge des Wassergehaltes des Chloroforms, und macht einer schwach bläulichen Platz. Zusatz von konzentrierter Schwefelsäure stellt die rote Farbe wieder her. In Neuberg (Analyse des Harns usw.>, S. 750 wird überhaupt zur Verdünnung der Pettenk offerschen Probe Schwefelsäure angegeben (Autor nicht genannt), jedenfalls muß reichlich Schwefelsäure vorhanden sein.

Hoppe-Seyler's Zeitschrift f. physiol. Chemie. XCVIII. 
7. Herr Prof. Hans Fischer hatte die Freundlichkeit, mich auf das äußerst charakteristische Verhalten des Natriumsalzes seiner Lithocholsäure aufmerksam zu machen, das nach seiner brieflichen Mitteilung am besten erhältlich ist, wenn man mit Natriumalkoholat in alkoholischer Lösung arbeitet. Das Natriumsalz bildet eine geleeartige Masse, die "sich in kürzester Zeit in haarförmige verfilzte Nadeln umwandelt. Dieses Verhalten konnte ich an der übersendeten Lithocholsäure mit frisch hergestelltem Natriumalkoholat im Überschuß ${ }^{1}$ ) durchans bestätigen, wenn auch nicht sofort, sondern erst nach passendem Eindampfen, dagegen gelang mir das mit meiner Säure bei wiederholten Versuchen nicht. Für weitere Untersuchungen reichte die kleine, von den Reaktionen noch übrig gebliebene Menge nicht aus. Es fragt sich nun, welche Gallensäure hier vorliegt. Um dem Leser sein Urteil zu erleichtern, sei auf die folgende kleine Tabelle hingewiesen, in welcher ich die wichtigsten Eigenschaften der nicht gepaarten Gallensäure zusammengestellt habe (siehe nebenstehende Tabelle).

Nach der Tabelle zu urteilen ist die Cholsäure ausgeschlossen wegen des negativen Ausfalles der so leicht anzustellenden und sicheren Jodreaktion von Mylius, es kann sich nur um Desoxycholsäure oder Lithocholsäure handeln, vielleicht auch Choleinsäure. Eine Entscheidung erlauben die bisherigen Beobachtungen nicht und es hat keinen Zweck, die Wahrscheinlichkeiten gegen einander abzuwägen, ich möchte nur bemerken, daß man für die Desoxycholsäure das gleichzeitige Vorkommen von stearinsäurehaltiger Palmitinsäure im Sinne von Wieland und Sorge geltend machen kann, das Verhalten der Säure aber mehr für Lithocholsäure spricht. Gegen diese spricht, daß das charakteristische Verhalten des Natriumsalzes nicht festgestellt werden konnte. Negative Resultate beweisen indessen nicht viel und Angaben über Löslichkeitsverhältnisse, die sich nur auf qualitative Beobachtungen gründen, sind $\mathrm{zu}$ subjektiver Natur, um entscheidend sein zu können. Die Verarbeitung eines größeren Materials wird hoffentlich Aufklärung bringen.

1) Ein gewisser Überschuß an Natriumalkoholat scheint erforderlich zu sein. 


\begin{tabular}{|c|c|c|c|c|c|}
\hline & Cholsäure & Choleinsäure & $\begin{array}{c}\text { Desoxychol- } \\
\text { säure }\end{array}$ & $\begin{array}{l}\text { Lithochol- } \\
\text { säure 1) }\end{array}$ & $\begin{array}{c}\text { Die von } \\
\text { mir erhaltene } \\
\text { Säure }\end{array}$ \\
\hline Schmelzpunkt & $195^{\circ}$ & $186-188^{\circ}$ & $\begin{array}{c}172-173^{\circ}, \\
\text { aus Eisessig } \\
144^{\circ}\end{array}$ & \begin{tabular}{|c|}
$184-186^{\circ}$ \\
aus $60^{\circ} \%$ iger \\
Essigsäure \\
\end{tabular} & $182^{\circ}$ \\
\hline Geschmack & $\begin{array}{c}\text { süßlich } \\
\text { bitter }\end{array}$ & rein bitter ${ }^{2}$ ) & $\begin{array}{c}\text { schwach } \\
\text { bitter }\end{array}$ & $\begin{array}{c}\text { geschmack- } \\
\text { los }\end{array}$ & geschmacklos \\
\hline Alkohol & leicht löslich & $\begin{array}{l}\text { heiß } 1: 5 \\
\text { kalt } 1: 8^{9} \text { ) }\end{array}$ & $\begin{array}{l}\text { heiß } 1: 2 \\
\left.\text { kalt } 1: 4^{3}\right)\end{array}$ & leicht löslich & leicht löslich \\
\hline Eisessig & leicht löslich & leicht löslich & $\begin{array}{l}\text { schwer } \\
\text { löslich }\end{array}$ & $\begin{array}{c}\text { mäßig } \\
\text { leicht löslich }\end{array}$ & $\begin{array}{c}\text { mäßig } \\
\text { leicht löslich }\end{array}$ \\
\hline $\begin{array}{l}\text { Pettenk ofer- } \\
\text { sche Probe mit } \\
\text { Alkohol verdünnt }\end{array}$ & $\begin{array}{l}\text { Absorptions- } \\
\text { streifen zw. } \\
\mathrm{D} \text { u.E u.bei } \mathrm{F} \\
\end{array}$ & & $\cdot$ & $\begin{array}{c}\text { Streifen bei } \\
\text { F stark }\end{array}$ & $\begin{array}{c}\text { Streifen bei } \\
\text { F stark }\end{array}$ \\
\hline $\begin{array}{c}\text { Jodreaktion } \\
\text { nach Mylius }\end{array}$ & positiv & negativ & negativ & negativ & negativ \\
\hline $\begin{array}{c}\text { Spezifische } \\
\text { Drehung }[\alpha]^{D}\end{array}$ & $\begin{array}{c}31,55^{\circ} \text { resp. } \\
37,02\end{array}$ & $48,47^{\circ}$ & $57,12^{\circ}$ & $32,14^{0}$ & $\begin{array}{c}\text { nicht } \\
\text { bestimmt }\end{array}$ \\
\hline
\end{tabular}

Auf die Bedeutung, die in der einen wie der anderen Säure infolge ihrer Schwerlöslichkeit für die Gallensteinbildung liegt, braucht nicht hingewiesen $\mathrm{zu}$ werden.

Bezüglich der Natur der in Versuch I beobachteten Gallensäure, die mit der früher aus Hundekot erhaltenen identisch zu sein scheint, enthalte ich mich jedes Urteils, da das Beobachtungsmaterial zu gering ist, nur Cholsäure ist auch hier ausgeschlossen.

Der Vollständigkeit wegen wurde auch in Versuch III das mit Äther erschöpfte Gallensteinpulver 10 Stunden im Soxhlet mit Alkohol absol. extrahiert. Der Alkoholauszug hinterließ beim Verdampfen 0,4944 g Rückstand (in Versuch I 0,4674,

1) Hans Fischer, Diese Zeitschr., Bd. 73, S. 234.

2) H. Fischer und P. Meyer fanden indessen ihre aus Rindergallensteinen isolierte Choleinsäure auch geschmacklos, Diese Zeitschr., Bd. 76, S. 96.

3) K. Wieland u. H. Sorge, Diese Zeitschr., Bd. 97, S. 1 u. ff. 
also ein sehr naheliegender Wert), dieser beim Veraschen 0,0228 Asche, aus der nur 0,0016 Calciumoxyd erhalten wurde.

\section{Zusammenfassung.}

1. Menschliche Gallensteine enthalten in geringer Menge freie stearinsäurehaltige Palmitinsäure. Das Vorkommen des Calciumsalzes derselben ist nicht ganz sicher erwiesen, jedenfalls seine Quantität noch kleiner, wie die der freien Säure.

2. Außer freier Palmitinsäure enthalten sie auch eine freie Gallensäure, über deren Natur - ob Desoxycholsäure oder die von Hans Fischer in Rindergallensteinen entdekte Lithocholsäure oder vielleicht auch Choleinsäure - eine bestimmte Entscheidung bisher nicht getroffen werden konnte.

3. Calciumpalmitat ist in Eisessig und Buttersäure leicht löslich und fällt beim Eingießen der Lösung in Wasser unverändert aus.

4. Cholesterin ist nicht nur in Eisessig, sondern auch in Buttersäure reichlich löslich. Die warme Lösung erstarrt beim Erkalten $\mathrm{zu}$ einem Brei von nadelförmigen Krystallen. Die Verbindung ist sehr locker, sie wird schon durch Alkohol zersetzt, es kann sich also nicht nur um molekulare Anlagerung handeln, analog dem essigsauren Cholesterin. 